\title{
TWO GENERIC RESULTS IN FIXED POINT THEORY
}

\author{
SIMEON REICH and ALEXANDER J. ZASLAVSKI \\ Department of Mathematics, The Technion-Israel Institute of Technology \\ 32000 Haifa, Israel \\ E-mail: sreich@tx.technion.ac.il,ajzasl@tx.technion.ac.il
}

\begin{abstract}
We give two examples of the generic approach to fixed point theory. The first example is concerned with the asymptotic behavior of infinite products of nonexpansive mappings in Banach spaces and the second with the existence and stability of fixed points of continuous mappings in finite-dimensional Euclidean spaces.
\end{abstract}

1. Introduction. The asymptotic behavior of infinite products of operators finds applications in many areas of Mathematics. See, for example, [1-5, 10-12, 14, 16-24] and the references mentioned therein. Given a bounded, closed and convex subset $K$ of a Banach space and a sequence $\mathbf{A}=\left\{A_{t}\right\}_{t=1}^{\infty}$ of self-mappings of $K$, we are interested in the convergence properties of the sequence of products $\left\{A_{n} \cdot \ldots \cdot A_{1} x\right\}_{n=1}^{\infty}$, where $x \in K$. In the special case of a constant sequence $\mathbf{A}$, we are led to study the asymptotic behavior of a single operator and the possible convergence of its powers to a fixed point. In their seminal 1976 paper [7], De Blasi and Myjak show that the powers of a generic nonexpansive self-mapping of $K$ do converge. Such an approach, when a certain property is investigated for a whole space of operators and not just for a single operator, has already been successfully applied in many areas of Analysis. For instance, in two recent papers $[18,22]$ we have extended the De Blasi-Myjak result in several directions to certain sequence spaces of nonexpansive mappings. One of these directions has involved weak ergodicity in the sense of population biology (see $[6,13,15,18,25]$ ). More precisely, we have shown that for most (in the sense of Baire category) sequences, the distances between the corresponding (random) infinite products with different initial points tend to zero, uniformly on $K$. The first main result of the present paper (Theorem 2.1 below) is a generic weak ergodic theorem for infinite products of nonself-mappings which takes into account computational errors. As a matter of fact, we use in this theorem the concept of

2000 Mathematics Subject Classification: 47H09, 47H10, 54E50, 54E52, 54H25.

Key words and phrases: Approximate fixed point, Banach space, complete metric space, Euclidean space, fixed point, generic property, infinite product, nonexpansive mapping, porous set, weak ergodic theorem, weakly inward.

The paper is in final form and no version of it will be published elsewhere. 
porosity $[8,9,24]$ which refines the notion of Baire's first category.

Our second and third main results (Theorems 3.1 and 3.2 below) establish generic existence and stability of fixed points for a class of nonself-mappings defined on certain closed (but not necessarily either convex or bounded) subsets of a finite-dimensional Euclidean space. In these theorems, we endow the relevant space of mappings with two topologies, one weaker than the other. In Theorem 3.1 we find an open (in the weak topology) and everywhere dense (in the strong topology) set such that each mapping in it possesses a fixed point. In Theorem 3.2 we construct a countable intersection of open (in the weak topology) and everywhere dense (in the strong topology) sets such that each mapping in this intersection has a stable fixed point.

2. Infinite products. Let $(X,\|\cdot\|)$ be a Banach space and let $K \subset X$ be a nonempty, bounded and closed subset of $X$.

Denote by $\mathcal{M}$ the set of all sequences $\left\{A_{t}\right\}_{t=1}^{\infty}$ such that each $A_{t}: K \rightarrow X, t=1,2, \ldots$, satisfies the following two conditions:

$$
\left\|A_{t} x-A_{t} y\right\| \leq\|x-y\| \text { for each } x, y \in K, \quad t=1,2, \ldots ;
$$

for any $\epsilon>0$, there exists a sequence $\left\{x_{t}\right\}_{t=0}^{\infty} \subset K$ such that $\left\|x_{t+1}-A_{t+1} x_{t}\right\| \leq \epsilon$, $t=0,1,2, \ldots$.

It is easy to see that if $K$ is a compact set in the norm topology, then for each $\left\{A_{t}\right\}_{t=1}^{\infty} \in \mathcal{M}$, there is $\left\{x_{t}\right\}_{t=0}^{\infty} \subset K$ such that $x_{t+1}=A_{t+1} x_{t}, t=0,1, \ldots$ Set

$$
\operatorname{rad}(K)=\sup \{\|x\|: x \in K\} .
$$

Proposition 2.1. Let $\left\{A_{t}\right\}_{t=1}^{\infty} \in \mathcal{M}$. Then for each integer $t \geq 1$ and each $x \in K$,

$$
\left\|A_{t} x\right\| \leq 3 \operatorname{rad}(K)+1 .
$$

Proof. By definition, there exists a sequence $\left\{x_{t}\right\}_{t=0}^{\infty} \subset K$ such that

$$
\left\|x_{t+1}-A_{t+1} x_{t}\right\| \leq 1, \quad t=0,1, \ldots
$$

By (2.1) and (2.3), for each integer $t \geq 0$ and each $x \in K$,

$$
\begin{aligned}
\left\|A_{t+1} x\right\| \leq\left\|A_{t+1} x-A_{t+1} x_{t}\right\|+\| A_{t+1} x_{t}- & x_{t+1}\|+\| x_{t+1} \| \\
& \leq\left\|x-x_{t}\right\|+1+\left\|x_{t+1}\right\| \leq 3 \operatorname{rad}(K)+1,
\end{aligned}
$$

as claimed.

For each $\left\{A_{t}\right\}_{t=1}^{\infty},\left\{B_{t}\right\}_{t=1}^{\infty} \in \mathcal{M}$, set

$$
\rho\left(\left\{A_{t}\right\}_{t=1}^{\infty},\left\{B_{t}\right\}_{t=1}^{\infty}\right)=\sup \left\{\left\|A_{t} x-B_{t} x\right\|: x \in K \text { and } t=1,2, \ldots\right\} .
$$

By Proposition 2.1, $\rho\left(\left\{A_{t}\right\}_{t=1}^{\infty},\left\{B_{t}\right\}_{t=1}^{\infty}\right)$ is finite for each pair $\left\{A_{t}\right\}_{t=1}^{\infty},\left\{B_{t}\right\}_{t=1}^{\infty} \in \mathcal{M}$. Clearly, $\rho$ is a metric on $\mathcal{M}$.

Proposition 2.2. The metric space $(\mathcal{M}, \rho)$ is complete.

Proof. Let $\left\{A_{t}^{(n)}\right\}_{t=1}^{\infty}, n=1,2, \ldots$, be a Cauchy sequence in $\mathcal{M}$. Clearly, for each $x \in K$ and each integer $t \geq 1$, the sequence $\left\{A_{t}^{(n)} x\right\}_{t=1}^{\infty}$ is a Cauchy sequence in $(X,\|\cdot\|)$ and therefore it converges to $A_{t} x \in X$ in the norm topology of $X$. Thus

$$
A_{t} x=\lim _{n \rightarrow \infty} A_{t}^{(n)} x \text { for each point } x \in K \text { and each integer } t \geq 1 .
$$


It is not difficult to see that for each integer $t \geq 1$,

$$
\left\|A_{t} x-A_{t} y\right\| \leq\|x-y\| \text { for all } x, y \in K \text {. }
$$

Let $\epsilon>0$. Since $\left\{A_{t}^{(n)}\right\}_{t=1}^{\infty}, n=1,2, \ldots$, is a Cauchy sequence, there exists a natural number $q$ such that for each pair of integers $m, n \geq q$,

$$
\left\|A_{t}^{(m)} x-A_{t}^{(n)} x\right\| \leq \epsilon / 8
$$

for all integers $t \geq 1$ and all points $x \in K$. By (2.7) and (2.5),

$$
\left\|A_{t}^{(m)} x-A_{t} x\right\| \leq \epsilon / 8
$$

for each integer $m \geq q$, each integer $t \geq 1$, and each $x \in K$. Since $\left\{A_{t}^{(q)}\right\}_{t=1}^{\infty} \in \mathcal{M}$, there is a sequence $\left\{x_{t}\right\}_{t=0}^{\infty} \subset K$ such that

$$
\left\|x_{t+1}-A_{t+1}^{(q)} x_{t}\right\| \leq \epsilon / 4, \quad t=0,1,2, \ldots
$$

In view of (2.9) and (2.8), for each integer $t \geq 0$,

$$
\left\|x_{t+1}-A_{t+1} x_{t}\right\| \leq\left\|x_{t+1}-A_{t+1}^{(q)} x_{t}\right\|+\left\|A_{t+1}^{(q)} x_{t}-A_{t+1} x_{t}\right\| \leq \epsilon / 4+\epsilon / 8<\epsilon / 2 .
$$

Since $\epsilon$ is an arbitrary positive number, we conclude that

$$
\left\{A_{t}\right\}_{t=1}^{\infty} \in \mathcal{M} .
$$

In view of $(2.8)$,

$$
\rho\left(\left\{A_{t}\right\}_{t=1}^{\infty},\left\{A_{t}^{(m)}\right\}_{t=1}^{\infty}\right) \leq \epsilon / 8 \text { for each integer } m \geq q .
$$

This completes the proof of Proposition 2.2.

Denote by $\mathcal{E}$ the set of all $\left\{A_{t}\right\}_{t=1}^{\infty} \in \mathcal{M}$ for which there exists a sequence $\left\{x_{t}\right\}_{t=1}^{\infty} \subset K$ such that

$$
A_{t+1} x_{t}=x_{t+1} \text { for all integers } t \geq 0 .
$$

Proposition 2.3. The set $\mathcal{E}$ is an everywhere dense subset of $\mathcal{M}$.

Proof. Let $\left\{A_{t}\right\}_{t=1}^{\infty} \in \mathcal{M}$ and $\epsilon>0$. By definition, there exists a sequence $\left\{x_{t}\right\}_{t=0}^{\infty} \subset K$ such that

$$
\left\|A_{t+1} x_{t}-x_{t+1}\right\| \leq \epsilon / 4, \quad t=0,1,2 \ldots
$$

For each $t=1,2, \ldots$, define

$$
B_{t} x=A_{t} x-A_{t} x_{t-1}+x_{t}, \quad x \in K .
$$

It is not difficult to see that

$$
B_{t} x_{t-1}=x_{t}, \quad t=1,2, \ldots, \quad\left\{B_{t}\right\}_{t=1}^{\infty} \in \mathcal{E}
$$

and

$$
\rho\left(\left\{A_{t}\right\}_{t=1}^{\infty},\left\{B_{t}\right\}_{t=1}^{\infty}\right) \leq \epsilon
$$

Proposition 2.3 is proved.

Before stating our first main result we recall the notion of porosity [8, 9, 24].

Let $(Y, d)$ be a complete metric space. We denote by $B(y, r)$ the closed ball of center $y \in Y$ and radius $r>0$. A subset $E \subset Y$ is called porous in $(Y, d)$ if there exist $\alpha \in(0,1)$ 
and $r_{0}>0$ such that for each $r \in\left(0, r_{0}\right]$ and each $y \in Y$, there exists $z \in Y$ for which

$$
B(z, \alpha r) \subset B(y, r) \backslash E .
$$

A subset of the space $Y$ is called $\sigma$-porous in $(Y, d)$ if it is a countable union of porous subsets in $(Y, d)$.

Since porous sets are nowhere dense, all $\sigma$-porous sets are of the first Baire category. If $Y$ is a finite-dimensional Euclidean space, then $\sigma$-porous sets are of Lebesgue measure zero. In fact, the class of $\sigma$-porous sets in such a space is much smaller than the class of sets which have measure zero and are of the first category.

TheOREM 2.1. There exists a set $\mathcal{F} \subset \mathcal{M}$ such that $\mathcal{M} \backslash \mathcal{F}$ is a $\sigma$-porous subset of $(\mathcal{M}, \rho)$ and such that for each $\left\{A_{t}\right\}_{t=1}^{\infty} \in \mathcal{F}$, the following property holds:

(P1) For each $\epsilon>0$, there exist $\delta>0$ and a natural number $T_{0}$ such that if the integers $m_{1}, m_{2}>T_{0}$ and if $\left\{x_{t}\right\}_{t=0}^{m_{1}},\left\{y_{t}\right\}_{t=0}^{m_{2}} \subset K$ satisfy

$$
\begin{aligned}
\left\|x_{t+1}-A_{t+1} x_{t}\right\| & \leq \delta, \quad t=0, \ldots, m_{1}-1, \\
\left\|y_{t+1}-A_{t+1} y_{t}\right\| & \leq \delta, \quad t=0, \ldots, m_{2}-1,
\end{aligned}
$$

then

$$
\left\|x_{t}-y_{t}\right\| \leq \epsilon, \quad t=T_{0}+1, \ldots, \min \left\{m_{1}, m_{2}\right\} .
$$

Proof. For each integer $n \geq 1$, denote by $\mathcal{F}_{n}$ the set of all $\left\{A_{t}\right\}_{t=1}^{\infty} \in \mathcal{M}$ for which the following property holds:

(P2) there exist $\delta>0$ and a natural number $T_{0}$ such that if the integers $m_{1}, m_{2}>T_{0}$ and $\left\{x_{t}\right\}_{t=0}^{m_{1}},\left\{y_{t}\right\}_{t=0}^{m_{2}} \subset K$ satisfy $(2.10)$, then

$$
\left\|x_{t}-y_{t}\right\| \leq 1 / n, \quad t=T_{0}+1, \ldots, \min \left\{m_{1}, m_{2}\right\} .
$$

Set

$$
\mathcal{F}=\bigcap_{n=1}^{\infty} \mathcal{F}_{n} .
$$

It is not difficult to see that for each $\left\{A_{t}\right\}_{t=1}^{\infty} \in \mathcal{F}$, property (P1) holds.

In order to complete the proof of the theorem, it is sufficient to show that for each integer $n \geq 1, \mathcal{M} \backslash \mathcal{F}_{n}$ is a porous subset of $(\mathcal{M}, \rho)$.

Indeed, let $n \geq 1$ be an integer. Choose a positive number

$$
\alpha<\left(2^{10} n\right)^{-1}(\operatorname{rad}(K)+1)^{-1} .
$$

Let $\left\{A_{t}\right\}_{t=1}^{\infty} \in \mathcal{M}$ and $r \in(0,1]$. By Proposition 2.3, there exists $\left\{B_{t}\right\}_{t=1}^{\infty} \in \mathcal{E}$ such that

$$
\rho\left(\left\{A_{t}\right\}_{t=1}^{\infty},\left\{B_{t}\right\}_{t=1}^{\infty}\right) \leq r / 16 .
$$

By the definition of $\mathcal{E}$, there exists $\left\{x_{t}\right\}_{t=0}^{\infty} \subset K$ such that

$$
B_{t+1} x_{t}=x_{t+1}, \quad t=0,1,2, \ldots
$$

Set

$$
\gamma=32 n \alpha r
$$

and let $t \geq 1$ be an integer. For each $x \in K$, set

$$
C_{t} x=\gamma x_{t}+(1-\gamma) B_{t} x
$$


It is easy to see that $\left\{C_{t}\right\}_{t=1}^{\infty} \in \mathcal{M}$ and that

$$
C_{t} x_{t-1}=x_{t}, \quad t=1,2 \ldots
$$

By (2.15), (2.2) and Proposition 2.1,

$$
\begin{aligned}
\rho\left(\left\{C_{t}\right\}_{t=1}^{\infty},\right. & \left.\left\{B_{t}\right\}_{t=1}^{\infty}\right) \leq \sup \left\{\left\|C_{t} z-B_{t} z\right\|: z \in K \text { and } t=1,2, \ldots\right\} \\
& =\sup \left\{\gamma\left\|x_{t}-B_{t} z\right\|: t=1,2, \ldots \text { and } z \in K\right\} \leq \gamma(4 \operatorname{rad}(K)+1) .
\end{aligned}
$$

Choose a natural number $T_{0}$ such that

$$
T_{0} \alpha r>\operatorname{rad}(K)+1
$$

and a positive number

$$
\delta<\min \left\{\alpha r,(8 n)^{-1} T_{0}^{-1}\right\} / 2 .
$$

Assume that

$$
\left\{D_{t}\right\}_{t=1}^{\infty} \in \mathcal{M} \quad \text { and } \quad \rho\left(\left\{D_{t}\right\}_{t=1}^{\infty},\left\{C_{t}\right\}_{t=1}^{\infty}\right) \leq \alpha r .
$$

By (2.20), (2.17), (2.13) and (2.15),

$$
\begin{array}{r}
\rho\left(\left\{D_{t}\right\}_{t=1}^{\infty},\left\{A_{t}\right\}_{t=1}^{\infty}\right) \\
\leq \rho\left(\left\{D_{t}\right\}_{t=1}^{\infty},\left\{C_{t}\right\}_{t=1}^{\infty}\right)+\rho\left(\left\{C_{t}\right\}_{t=1}^{\infty},\left\{B_{t}\right\}_{t=1}^{\infty}\right)+\rho\left(\left\{B_{t}\right\}_{t=1}^{\infty},\left\{A_{t}\right\}_{t=1}^{\infty}\right) \\
\leq \alpha r+4 \gamma(\operatorname{rad}(K)+1)+r / 16 \leq \alpha r+32 n \alpha r \cdot(4 \operatorname{rad}(K)+1)+r / 16 \\
\leq r[8 \cdot 32 n \alpha(\operatorname{rad}(K)+1)+1 / 16] \leq r / 2 .
\end{array}
$$

Thus

$$
\rho\left(\left\{D_{t}\right\}_{t=1}^{\infty},\left\{A_{t}\right\}_{t=1}^{\infty}\right) \leq r / 2
$$

Assume that the integers $m_{1}, m_{2}>T_{0}$ and that $\left\{x_{t}\right\}_{t=0}^{m_{1}},\left\{y_{t}\right\}_{t=0}^{m_{2}} \subset K$ satisfy

$$
\begin{aligned}
\left\|x_{t+1}-D_{t+1} x_{t}\right\| & \leq \delta, \quad t=0, \ldots, m_{1}-1 \\
\left\|y_{t+1}-D_{t+1} y_{t}\right\| & \leq \delta, \quad t=0, \ldots, m_{2}-1 .
\end{aligned}
$$

We now show that

$$
\left\|x_{t}-y_{t}\right\| \leq 1 / n, \quad t=T_{0}+1, \ldots, \min \left\{m_{1}, m_{2}\right\} .
$$

Indeed, let an integer $T$ satisfy

$$
T_{0}+1 \leq T \leq \min \left\{m_{1}, m_{2}\right\}
$$

We claim that there is an integer $j \in\left\{T-T_{0}, \ldots, T-1\right\}$ such that

$$
\left\|x_{j}-y_{j}\right\| \leq(4 n)^{-1} \text {. }
$$

Let us assume the converse. Then for each $j=T-T_{0}, \ldots, T-1$,

$$
\left\|x_{j}-y_{j}\right\|>(4 n)^{-1} \text {. }
$$


It follows from $(2.22),(2.20),(2.25)$ and $(2.1)$ that for $j=T-T_{0}, \ldots, T-1$,

$$
\begin{aligned}
\left\|x_{j+1}-y_{j+1}\right\| & \leq\left\|x_{j+1}-D_{j+1} x_{j}\right\|+\left\|D_{j+1} x_{j}-D_{j+1} y_{j}\right\|+\left\|D_{j+1} y_{j}-y_{j+1}\right\| \\
& \leq \delta+\left\|D_{j+1} x_{j}-D_{j+1} y_{j}\right\|+\delta \\
& \leq 2 \delta+2 \rho\left(\left\{D_{t}\right\}_{t=1}^{\infty},\left\{C_{t}\right\}_{t=1}^{\infty}\right)+\left\|C_{j+1} x_{j}-C_{j+1} y_{j}\right\| \\
& \leq 2 \delta+2 \alpha r+(1-\gamma)\left\|B_{j+1} x_{j}-B_{j+1} y_{j}\right\| \\
& \leq 2 \delta+2 \alpha r+(1-\gamma)\left\|x_{j}-y_{j}\right\| .
\end{aligned}
$$

By this inequality, (2.26), (2.19) and (2.15),

$$
\left\|x_{j}-y_{j}\right\|-\left\|x_{j+1}-y_{j+1}\right\| \geq \gamma\left\|x_{j}-y_{j}\right\|-2 \delta-2 \alpha r \geq \gamma(4 n)^{-1}-4 \alpha r \geq 4 \alpha r .
$$

Together with (2.2) and (2.18), this implies that

$$
2 \operatorname{rad}(K) \geq\left\|x_{T-T_{0}}-y_{T-T_{0}}\right\|-\left\|x_{T}-y_{T}\right\| \geq T_{0} 4 \alpha r>4 \operatorname{rad}(K)+4 .
$$

The contradiction we have reached demonstrates that indeed there is an integer $j \in\left\{T-T_{0}, \ldots, T-1\right\}$ such that $(2.25)$ holds.

By (2.1) and (2.22), for each integer $i$ satisfying $j \leq i \leq T-1$,

$$
\begin{array}{r}
\left\|x_{i+1}-y_{i+1}\right\| \leq\left\|x_{i+1}-D_{i+1} x_{i}\right\|+\left\|D_{i+1} x_{i}-D_{i+1} y_{i}\right\|+\left\|D_{i+1} y_{i}-y_{i+1}\right\| \\
\leq \delta+\left\|x_{i}-y_{i}\right\|+\delta .
\end{array}
$$

When combined with (2.25) and (2.19), this relation implies that

$$
\left\|x_{T}-y_{T}\right\| \leq\left\|x_{j}-y_{j}\right\|+2 \delta(T-j) \leq\left\|x_{j}-y_{j}\right\|+2 \delta T_{0} \leq(4 n)^{-1}+(4 n)^{-1} .
$$

Therefore

$$
\left\|x_{T}-y_{T}\right\| \leq(2 n)^{-1}
$$

for all integers $T$ satisfying (2.24). Thus we have shown that each sequence $\left\{D_{t}\right\}_{t=1}^{\infty} \in \mathcal{M}$ satisfying (2.20) possesses property (P2) and consequently belongs to $\mathcal{F}_{n}$. Therefore $\mathcal{M} \backslash \mathcal{F}_{n}$ is indeed a porous subset of $(\mathcal{M}, \rho)$. This completes the proof of Theorem 2.1.

3. Existence and stability. Let $K \subset R^{n}$ be a nonempty, closed subset of the $n$ dimensional Euclidean space $\left(R^{n},\|\cdot\|\right)$. We suppose that $K$ is the closure of its nonempty interior $\operatorname{int}(K)$.

For each $x \in R^{n}$ and each $r>0$, set $B(x, r)=\left\{y \in R^{n}:\|x-y\| \leq r\right\}$ and fix $\theta \in K$.

Denote by $\mathcal{M}$ the set of all continuous mappings $A: K \rightarrow R^{n}$. We equip the space $\mathcal{M}$ with the uniformity determined by the base

$$
\mathcal{E}_{w}(N, \epsilon)=\{(A, B) \in \mathcal{M} \times \mathcal{M}:\|A x-B x\| \leq \epsilon \text { for all } x \in B(\theta, N) \cap K\},
$$

where $N, \epsilon>0$.

Clearly, the space $\mathcal{M}$ with this uniformity is metrizable and complete. We equip the space $\mathcal{M}$ with the topology induced by this uniformity. This topology will be called the weak topology.

We also equip the space $\mathcal{M}$ with the uniformity determined by the base

$$
\mathcal{E}_{s}(\epsilon)=\{(A, B) \in \mathcal{M} \times \mathcal{M}:\|A x-B x\| \leq \epsilon \text { for all } x \in K\},
$$


where $\epsilon>0$. Clearly, the space $\mathcal{M}$ with this uniformity is also metrizable and complete. The topology induced by this uniformity on $\mathcal{M}$ will be called the strong topology.

Denote by $\mathcal{M}_{f}$ the set of all $A \in \mathcal{M}$ which have approximate fixed points. In other words, the set $\mathcal{M}_{f}$ consists of all $A \in \mathcal{M}$ such that

$$
\inf \{\|x-A x\|: x \in K\}=0 .
$$

It is clear that $\mathcal{M}_{f}$ is a closed subset of $\mathcal{M}$ with the strong topology.

Note that if the set $K$ is bounded, then $\mathcal{M}_{f}$ consists of all those elements of $\mathcal{M}$ which have fixed points. Every self-mapping of $K$ which is a strict contraction, that is, has a Lipschitz constant strictly less than one, clearly belongs to $\mathcal{M}_{f}$.

If $K$ is bounded and convex, and a continuous mapping $A: K \rightarrow R^{n}$ satisfies the Leray-Schauder condition with respect to $w \in \operatorname{int}(K)$, that is, $A y-w \neq m(y-w)$ for all $y$ on the boundary of $K$ and $m>1$, then it also belongs to $\mathcal{M}_{f}$. If such an $A$ is a strict contraction, then this continues to be true even if $K$ is neither bounded nor convex.

We endow the topological subspace $\mathcal{M}_{f} \subset M$ with both the relative weak and strong topologies.

THEOREM 3.1. Let $\gamma \in(0,1)$. There exists an open (in the weak topology), everywhere dense (in the strong topology) set $\mathcal{F}_{\gamma} \subset \mathcal{M}_{f}$ such that for each $A \in \mathcal{F}_{\gamma}$, there are $x_{A} \in \operatorname{int}(K), r_{A} \in(0,1)$, and a neighborhood $\mathcal{U}$ of $A$ in $\mathcal{M}_{f}$ with the weak topology such that

$$
B\left(x_{A}, r_{A}\right) \subset K \quad \text { and } \quad A x_{A}=x_{A},
$$

and for each $C \in \mathcal{U}$, there is $x_{C} \in K$ such that $C x_{C}=x_{C}$ and $\left\|x_{C}-x_{A}\right\| \leq \gamma r_{A}$.

THEOREM 3.2. There exists a set $\mathcal{F} \subset \mathcal{M}_{f}$ which is a countable intersection of open (in the weak topology), everywhere dense (in the strong topology) subsets of $\mathcal{M}_{f}$ such that for each $A \in \mathcal{F}$ and each $\gamma \in(0,1)$, there exist $x_{A} \in \operatorname{int}(K), r_{A} \in(0,1)$, and a neighborhood $\mathcal{U}$ of $A$ in $\mathcal{M}_{f}$ with the weak topology such that

$$
B\left(x_{A}, r_{A}\right) \subset K \quad \text { and } \quad A x_{A}=x_{A},
$$

and for each $C \in \mathcal{U}$, there is $x_{C} \in K$ such that $C x_{C}=x_{C}$ and $\left\|x_{C}-x_{A}\right\| \leq \gamma r_{A}$.

ExAmple. Let $n=1, K=\bigcup_{j=0}^{\infty}[2 j, 2 j+1]$, and define, for each integer $j \geq 1$ and each $x \in[2 j, 2 j+1], A x=x+2^{-j}$. Clearly, $\inf \{|x-A x|: x \in K\}=0$, but $A$ is fixed point free.

4. Auxiliary results. Denote by $\mathcal{E}$ the set of all $A \in \mathcal{M}_{f}$ for which there exist

$$
x_{A} \in \operatorname{int}(K) \text { and } r_{A} \in(0,1)
$$

such that

$$
B\left(x_{A}, r_{A}\right) \subset K \text { and } A y=x_{A} \text { for all } y \in B\left(x_{A}, r_{A} / 4\right) .
$$

Lemma 4.1. The set $\mathcal{E}$ is an everywhere dense subset of $\mathcal{M}_{f}$ with the strong topology.

Proof. Let $A \in \mathcal{M}_{f}$ and $\epsilon>0$. By the definition of $\mathcal{M}_{f}$ (see (3.3)), there exists $x_{0} \in K$ such that

$$
\left\|A x_{0}-x_{0}\right\|<\epsilon / 16 \text {. }
$$


Since $K$ is the closure of $\operatorname{int}(K)$ and $A$ is continuous, there is $x_{1} \in \operatorname{int}(K)$ such that

$$
\left\|x_{1}-x_{0}\right\|<\epsilon / 16 \text { and }\left\|A x_{1}-A x_{0}\right\|<\epsilon / 16 .
$$

Set

$$
A_{1} y=A y-A x_{1}+x_{1}, \quad y \in K .
$$

Clearly, $A_{1} \in \mathcal{M}$. In view of (4.5),

$$
A_{1} x_{1}=x_{1} .
$$

By (4.5), (4.4) and (4.3), for each $y \in K$,

$$
\left\|A y-A_{1} y\right\|=\left\|A x_{1}-x_{1}\right\| \leq\left\|A x_{1}-A x_{0}\right\|+\left\|A x_{0}-x_{0}\right\|+\left\|x_{0}-x_{1}\right\|<3 \epsilon / 16 .
$$

Since $A_{1}$ has a fixed point (see (4.6)), it is clear that $A_{1} \in \mathcal{M}_{f}$. Since $A_{1}$ is continuous and $x_{1} \in \operatorname{int}(K)$, there exists $r_{1} \in(0,1)$ such that

$$
B\left(x_{1}, r_{1}\right) \subset K \quad \text { and } \quad\left\|A_{1} x-A_{1} x_{1}\right\| \leq \epsilon / 16 \text { for all } x \in B\left(x_{1}, r_{1}\right) .
$$

Define

$$
\psi(t)= \begin{cases}1, & t \in\left[0, r_{1} / 2\right], \\ 2\left(r_{1}-t\right) r_{1}^{-1}, & t \in\left(r_{1} / 2, r_{1}\right), \\ 0, & t \in\left[r_{1}, \infty\right),\end{cases}
$$

and

$$
B y=\psi\left(\left\|y-x_{1}\right\|\right) x_{1}+\left(1-\psi\left(\left\|y-x_{1}\right\|\right)\right) A_{1} y, \quad y \in K .
$$

Clearly, $B \in \mathcal{M}$. It follows from (4.10) and (4.9) that for each $y \in B\left(x_{1}, r_{1} / 2\right)$,

$$
B y=x_{1} .
$$

Therefore $B \in \mathcal{E}$. We will now show that

$$
\|B y-A y\| \leq \epsilon \text { for all } x \in K .
$$

Indeed, let $y \in K$. There are two cases to be considered:

$$
\begin{gathered}
\left\|x_{1}-y\right\| \leq r_{1} ; \\
\left\|x_{1}-y\right\|>r_{1} .
\end{gathered}
$$

If (4.13) holds, then (4.13), (4.10), (4.9) and (4.7) imply that

$$
B y=A_{1} y \quad \text { and } \quad\|B y-A y\|=\left\|A_{1} y-A y\right\|<\epsilon / 4 .
$$

Let (4.12) hold. Then by (4.12), (4.10), (4.9), (4.6) and (4.8),

$$
\left\|B y-A_{1} y\right\|=\left\|\psi\left(\left\|y-x_{1}\right\|\right)\left(x_{1}-A_{1} y\right)\right\| \leq\left\|x_{1}-A_{1} y\right\|=\left\|A_{1} x_{1}-A_{1} y\right\|<\epsilon / 16 .
$$

When combined with (4.7), this inequality implies that

$$
\|B y-A y\| \leq\left\|B y-A_{1} y\right\|+\left\|A_{1} y-A y\right\| \leq \epsilon / 16+3 \epsilon / 16=\epsilon / 4 .
$$

Thus

$$
\|B y-A y\| \leq \epsilon / 4 \text { for all } y \in K .
$$

This completes the proof of Lemma 4.1 . 
Lemma 4.2. Let $A \in \mathcal{E}, x_{A} \in \operatorname{int}(K), r_{A} \in(0,1)$ satisfy (4.2), and let $\gamma \in(0,1)$. Then there exists a neighborhood $\mathcal{U}$ of $A$ in $\mathcal{M}_{f}$ with the weak topology such that for each $B \in \mathcal{U}$, there is $x_{B} \in K$ such that $\left\|x_{B}-x_{A}\right\| \leq \gamma r_{A} / 4$ and $B x_{B}=x_{B}$.

Proof. Set

$$
\Delta=\gamma r_{A} / 4
$$

and put

$$
\mathcal{U}=\left\{B \in \mathcal{M}_{f}:\|B z-A z\| \leq \Delta \text { for each } z \in B\left(x_{A}, r_{A}\right)\right\} .
$$

Clearly, $\mathcal{U}$ is a neighborhood of $A$ in $\mathcal{M}_{f}$ with the weak topology.

Let $B \in \mathcal{U}$. It follows from (4.16), (4.2) and (4.15) that for each $z \in B\left(x_{A}, \gamma r_{A} / 4\right)$,

$$
\left\|B z-x_{A}\right\| \leq\|B z-A z\|+\left\|A z-x_{A}\right\| \leq \Delta+\left\|A z-x_{A}\right\|=\Delta=\gamma r_{A} / 4 .
$$

Thus

$$
B\left(B\left(x_{A}, \gamma r_{A} / 4\right)\right) \subset B\left(x_{A}, \gamma r_{A} / 4\right)
$$

Since the mapping $B$ is continuous, there is $x_{B} \in B\left(x_{A}, \gamma r_{A} / 4\right)$ such that

$$
B x_{B}=x_{B} \text {. }
$$

Lemma 4.2 is proved.

\section{Proofs of Theorems 3.1 and 3.2}

Proof of Theorem 3.1. Let $A \in \mathcal{E}$. There exist $x_{A} \in \operatorname{int}(K)$ and $r_{A} \in(0,1)$ such that (4.2) holds. By Lemma 4.2, there exists an open neighborhood $\mathcal{U}(A)$ of $A$ in $\mathcal{M}_{f}$ with the weak topology such that the following property holds:

(P3) For each $B \in \mathcal{U}(A)$, there is $x_{B} \in K$ such that

$$
B x_{B}=x_{B} \text { and } \quad\left\|x_{B}-x_{A}\right\| \leq \gamma r_{A} / 8 .
$$

Set

$$
\mathcal{F}_{\gamma}=\bigcup\{\mathcal{U}(A): A \in \mathcal{E}\} .
$$

By Lemma $4.1, \mathcal{F}_{\gamma}$ is an open (in the weak topology), everywhere dense (in the strong topology) subset of $\mathcal{M}_{f}$.

Let $B \in \mathcal{F}_{\gamma}$. By (5.2), there is $A \in \mathcal{E}$ such that

$$
B \in \mathcal{U}(A) \text {. }
$$

By property (P3), for each $C \in \mathcal{U}(A)$, there is $x_{C} \in K$ such that

$$
C x_{C}=x_{C} \text { and }\left\|x_{C}-x_{A}\right\| \leq \gamma r_{A} / 8 \text {. }
$$

Clearly,

$$
\left\|x_{B}-x_{A}\right\| \leq \gamma r_{A} / 8
$$

It follows from (5.5) and (4.2) that

$$
B\left(x_{B}, r_{A} / 2\right) \subset B\left(x_{A}, r_{A}\right) \subset K .
$$

By (5.4) and (5.5), for each $C \in \mathcal{U}(A)$,

$$
\left\|x_{C}-x_{B}\right\| \leq\left\|x_{C}-x_{A}\right\|+\left\|x_{A}-x_{B}\right\| \leq \gamma r_{A} / 8+\gamma r_{A} / 8=\gamma r_{A} / 4 .
$$

This completes the proof of Theorem 3.1. 
Proof of Theorem 3.2. For each integer $n \geq 1$, let $\mathcal{F}_{n}$ be as guaranteed in Theorem 3.1 with $\gamma=(2 n)^{-1}$. Set

$$
\mathcal{F}=\bigcap_{n=1}^{\infty} \mathcal{F}_{n} .
$$

Clearly, $\mathcal{F}$ is a countable intersection of open (in the weak topology), everywhere dense (in the strong topology) subsets of $\mathcal{M}_{f}$.

Let $A \in \mathcal{F}$ and $\gamma \in(0,1)$. Choose a natural number $n$ such that

$$
n^{-1}<\gamma / 8
$$

Since $A \in \mathcal{F}_{n}$ and the assertion of Theorem 3.1 holds with $\gamma=(2 n)^{-1}$ and $\mathcal{F}_{\gamma}=\mathcal{F}_{n}$, there are $x_{A} \in \operatorname{int}(K), r_{A} \in(0,1)$, and a neighborhood $\mathcal{U}$ of $A$ in $\mathcal{M}_{f}$ with the weak topology such that $B\left(x_{A}, r_{A}\right) \subset K, A x_{A}=x_{A}$, and for each $C \in \mathcal{U}$ there is $x_{C} \in K$ such that $C x_{C}=x_{C}$ and

$$
\left\|x_{C}-x_{A}\right\| \leq r_{A}(2 n)^{-1}<r_{A} \gamma .
$$

Thus Theorem 3.2 is also established.

Acknowledgments. The first author was partially supported by the Fund for the Promotion of Research at the Technion and by the Technion VPR Fund. He also thanks the Erwin Schrödinger International Institute for Mathematical Physics in Vienna for its support.

\section{References}

[1] I. Amemiya, T. Ando, Convergence of random products of contractions in Hilbert space, Acta Sci. Math. (Szeged) 26 (1965), 239-244.

[2] H. H. Bauschke, A norm convergence result on random products of relaxed projections in Hilbert space, Trans. Amer. Math. Soc. 347 (1995), 1365-1373.

[3] H. H. Bauschke, J. M. Borwein, On projection algorithms for solving convex feasibility problems, SIAM Rev. 38 (1996), 367-426.

[4] H. H. Bauschke, J. M. Borwein, A. S. Lewis, The method of cyclic projections for closed convex sets in Hilbert space, in: Recent Developments in Optimization Theory and Nonlinear Analysis (Jerusalem 1995), Contemp. Math. 204, Amer. Math. Soc., Providence 1997, 1-38.

[5] H. Brézis, P.-L. Lions, Produits infinis de résolvantes, Israel J. Math. 29 (1978), 329-345.

[6] J. E. Cohen, Ergodic theorems in demography, Bull. Amer. Math. Soc. (N.S.) 1 (1979), 275-295.

[7] F. S. De Blasi, J. Myjak, Sur la convergence des approximations successives pour les contractions non linéaires dans un espace de Banach, C. R. Acad. Sci. Paris Sér. A-B 283 (1976), A185-A187.

[8] F. S. De Blasi, J. Myjak, Sur la porosité de l'ensemble des contractions sans point fixe, C. R. Acad. Sci. Paris Sér. I Math. 308 (1989), 51-54

[9] F. S. De Blasi, J. Myjak, P. L. Papini, Porous sets in best approximation theory, J. London Math. Soc. (2) 44 (1991), 135-142.

[10] J. Dye, M. A. Khamsi, S. Reich, Random products of contractions in Banach spaces, Trans. Amer. Math. Soc. 325 (1991), 87-99. 
[11] J. Dye, T. Kuczumow, P.-K. Lin, S. Reich, Convergence of unrestricted products of nonexpansive mappings in spaces with the Opial property, Nonlinear Anal. 26 (1996), 767-773.

[12] J. Dye, S. Reich, Random products of nonexpansive mappings, in: Optimization and Nonlinear Analysis (Haifa 1990), Pitman Res. Notes Math. Ser. 244, Longman Sci. Tech., Harlow 1992, 106-118.

[13] T. Fujimoto, U. Krause, Asymptotic properties for inhomogeneous iterations of nonlinear operators, SIAM J. Math. Anal. 19 (1988), 841-853.

[14] P.-K. Lin, Unrestricted products of contractions in Banach spaces, Nonlinear Anal. 24 (1995), 1103-1108.

[15] R. D. Nussbaum, Some nonlinear weak ergodic theorems, SIAM J. Math. Anal. 21 (1990), 436-460.

[16] S. Reich, The alternating algorithm of von Neumann in the Hilbert ball, Dynam. Systems Appl. 2 (1993), 21-25.

[17] S. Reich, I. Shafrir, Nonexpansive iterations in hyperbolic spaces, Nonlinear Anal. 15 (1990), $537-558$.

[18] S. Reich, A. J. Zaslavski, Convergence of generic infinite products of nonexpansive and uniformly continuous operators, Nonlinear Anal. 36 (1999), 1049-1065.

[19] S. Reich, A. J. Zaslavski, Convergence of generic infinite products of order-preserving mappings, Positivity 3 (1999), 1-21.

[20] S. Reich, A. J. Zaslavski, Convergence of Krasnoselskii-Mann iterations of nonexpansive operators, Math. Comput. Modelling 32 (2000), 1423-1431.

[21] S. Reich, A. J. Zaslavski, Infinite products of resolvents of accretive operators, Topol. Methods Nonlinear Anal. 15 (2000), 153-168.

[22] S. Reich, A. J. Zaslavski, Generic convergence of infinite products, J. Nonlinear Convex Anal. 2 (2001), 111-127.

[23] S. Reich, A. J. Zaslavski, Generic convergence of infinite products of nonexpansive mappings in Banach and hyperbolic spaces, in: Optimization and Related Topics (Ballarat 1999), Appl. Optim. 47, Kluwer, Dordrecht 2001, 371-402.

[24] S. Reich, A. J. Zaslavski, The set of divergent infinite products in a Banach space is $\sigma$-porous, Z. Anal. Anwendungen 21 (2002), 865-878.

[25] S. Reich, A. J. Zaslavski, A weak ergodic theorem for infinite products of Lipschitzian mappings, Abstr. Appl. Anal. 2003, no. 2, 67-74. 
